\title{
Operations on hyperideals in ordered Krasner hyperrings
}

\author{
S. Omidi, B. Davvaz and P. Corsini
}

\begin{abstract}
In the present paper, we will concentrate our efforts on ordered Krasner hyperrings and investigate some of their related properties. Moreover, we introduce and analyze the notion of interior hyperideal in ordered Krasner hyperrings. We also characterize intra-regular ordered Krasner hyperrings by the properties of these interior hyperideals. Finally, we give some results on ordered Krasner hyperrings.
\end{abstract}

\section{Introduction}

Algebraic hyperstructures are a generalization of classical algebraic structures. In a classical algebraic structure, the composition of two elements of a set is again an element of the same set, while in an algebraic hyperstructure, the composition of two elements is a non-empty subset of the same set. The theory of hyperstructures has been initiated in 1934 by a French mathematician, Marty [24], during the 8th Congress of Scandinavian Mathematicians. Marty introduced hypergroups as a generalization of groups. The hyperstructure theory and its applications have been investigated by the contribution of many mathematicians. The principal notions of hyperstructure theory can be found in $[9,10,11,12,14,29]$.

A semigroup is an algebraic structure consisting of a non-empty set $S$ together with an associative binary operation. A semigroup $(S, \cdot)$ is called an

Key Words: Algebraic hyperstructure, Ordered Krasner hyperring, Hyperideal, Interior hyperideal, Intra-regular.

2010 Mathematics Subject Classification: Primary 16Y99; Secondary 20N20.

Received: 18.05 .2015

Revised: 25.06 .2015

Accepted: 30.06 .2015 
ordered semigroup [5] if there is a partial order $\leq$ on $S$ such that $a \leq b$ implies $x \cdot a \leq x \cdot b$ and $a \cdot x \leq b \cdot x$ for any $a, b, x \in S$. The notion of interior ideal of an ordered semigroup has been introduced by Kehayopulu in $[20]$. Let $(S, \cdot, \leq)$ be an ordered semigroup. A subsemigroup $A$ of $S$ is called an interior ideal of $S$ if (1) $S A S \subseteq A$ and (2) If $x \in A, y \in S$ and $y \leq x$, then $y \in A$. In regular and intra-regular ordered semigroups the concepts of ideals and interior ideals coincide. Let $(S, \cdot, \leq)$ be an ordered semigroup. A non-empty subset $A$ of $S$ is called a left (resp. right) ideal of $S$ if (1) $S A \subseteq A$ (resp. $A S \subseteq A$ ) and (2) If $x \in A$ and $y \in S$ such that $y \leq x$, then $y \in A$. Good and Hughes [16] introduced the notion of bi-ideals of a semigroup as a generalization of left (right) ideals. We mean by a $b i$-ideal is a subsemigroup $A$ of a $\operatorname{semigroup~}(S, \cdot)$ such that $A S A \subseteq A$. Prime bi-ideals, strongly prime bi-ideals and semiprime bi-ideals in a semigroup were discussed by Shabir and Kanwal in [27]. Kehayopulu et al. [21] characterized the intra-regular ordered semigroups in terms of right ideals and left ideals of ordered semigroups.

The concept of a semihypergroup is a generalization of the concept of a semigroup. Many authors studied different aspects of semihypergroups. In [17], Heidari and Davvaz studied a semihypergroup $(H, \circ)$ besides a binary relation $\leq$, where $\leq$ is a partial order relation such that satisfies the monotone conditin. An ordered semihypergroup $(S, \circ, \leq)$ is a semihypergroup $(S, \circ)$ together with a partial order $\leq$ that is compatible with the hyperoperation, meaning that for any $x, y, z$ in $S$,

$$
x \leq y \Rightarrow z \circ x \leq z \circ y \text { and } x \circ z \leq y \circ z .
$$

Here, $z \circ x \leq z \circ y$ means for any $a \in z \circ x$ there exists $b \in z \circ y$ such that $a \leq b$. The case $x \circ z \leq y \circ z$ is defined similarly. In [6], Changphas and Davvaz studied some properties of hyperideals in ordered semihypergroups. Ordered polygroups was introduced in a paper of Bakhshi and Borzooei [4]. The concept of ordering hypergroups introduced by Chvalina [7] as a special class of hypergroups and studied by many authors, for example, Chvalina [7], Chvalina and Moucka [8], Davvaz et al. [15], Hoskova [18, 19].

The aim of this paper is to give some results on ordered Krasner hyperrings. The structure of the paper is organized as follows: In Section 2, we discuss some basic concepts of Krasner hyperrings. We recall some elementary definitions and results concerning Krasner hyperrings, which we need for development of our paper. The reader is referred to [14] for the notions and notations of hyperring theory. Section 3 is devoted to characterize the several properties of ordered Krasner hyperrings. In Section 4 of this paper, we introduce interior hyperideals in ordered Krasner hyperrings and investigate some related properties. Moreover, we consider characterizations of ordered Krasner hyperrings which are intra-regular. In Section 5, we give some results 
on ordered Krasner hyperrings.

\section{Terminology and basic properties}

In this section, we provide all the background notations and definitions will be used throughout this paper.

Definition 2.1. [25] A canonical hypergroup is a non-empty set $H$ endowed with an additive hyperoperation $+: H \times H \rightarrow \mathcal{P}^{*}(H)$, satisfying the following properties:

(1) for any $x, y, z \in H, x+(y+z)=(x+y)+z$,

(2) for any $x, y \in H, x+y=y+x$,

(3) there exists $0 \in H$ such that $0+x=x$, for any $x \in H$,

(4) for every $x \in H$, there exists a unique element $x^{\prime} \in H$ such that $0 \in x+x^{\prime}$ (we shall write $-x$ for $x^{\prime}$ and we call it the opposite of $x$ ),

(5) $z \in x+y$ implies that $y \in-x+z$ and $x \in z-y$, that is $(H,+)$ is reversible.

The following equalities follow easily from the axioms: (i) $-(-a)=a$; (ii) $a+H=H$ for all $a \in H$; and (iii) $-(a+b)=-a-b$ for all $a, b \in H$.

Krasner has studied the notion of hyperfields and hyperring in [22]. Some authors, namely, Davvaz [13], Nakassis [26], Spartalis [28] and others followed him. Hyperrings are essentially rings, with approximately modified axioms in which addition is a hyperoperation. Let us survey some definitions and results on Krasner hyperrings such that we will apply in the next sections.

Definition 2.2. [22] A Krasner hyperring is an algebraic hyperstructure $(R,+, \cdot)$ which satisfies the following axioms:

(1) $(R,+)$ is a canonical hypergroup,

$(2)(R, \cdot)$ is a semigroup having zero as a bilaterally absorbing element, i.e., $x \cdot 0=0 \cdot x=0$,

(3) The multiplication is distributive with respect to the hyperoperation + .

We call 0 the zero of the Krasner hyperring $(R,+, \cdot)$. For $x \in R$, let $-x$ denote the unique inverse of $x$ in $(R,+)$. Then $-(-x)=x$, for all $x \in R$. In addition, $(x+y) \cdot(z+w) \subseteq x \cdot z+x \cdot w+y \cdot z+y \cdot w,(-x) \cdot y=x \cdot(-y)=-(x \cdot y)$, for every $x, y, z, w \in R$. 
A Krasner hyperring $R$ is called commutative (with unit element) if $(R, \cdot)$ is a commutative semigroup (with unit element). A Krasner hyperfield is a Krasner hyperring for which $(R-\{0\}, \cdot)$ is a group. A non-empty subset $A$ of a canonical hypergroup $H$ is called a subcanonical hypergroup of $H$ if $A$ is a canonical hypergroup under the hyperoperation of $H$. Let $(H,+)$ be a canonical hypergroup and $A \subseteq H$. Then $A$ is a subcanonical hypergroup of $H$ if and only if $x-y \subseteq A$ for all $x, y \in A$. A subhyperring of a Krasner hyper$\operatorname{ring}(R,+, \cdot)$ is a non-empty subset $A$ of $R$ which forms a Krasner hyperring containing 0 under the hyperoperation + and the operation $\cdot$ on $R$, that is, $A$ is a canonical subhypergroup of $(R,+)$ and $A \cdot A \subseteq A$. Then a non-empty subset $A$ of $R$ is a subhyperring of $(R,+, \cdot)$ if and only if, for all $x, y \in A$, $x+y \subseteq A,-x \in A$ and $x \cdot y \in A$. So, a non-empty subset $A$ of $R$ is called a subhyperring of $R$ if $x-y \subseteq A$ and $x \cdot y \in A$ for all $x, y \in A$.

Definition 2.3. [9] A non-empty subset $I$ of a Krasner hyperring $(R,+, \cdot)$ is called a left (resp. right) hyperideal of $R$ if $(I,+)$ is a canonical subhypergroup of $(R,+)$ and for every $a \in I$ and $r \in R, r \cdot a \in I$ (resp. $a \cdot r \in I$ ). A hyperideal of $(R,+, \cdot)$ is one which is a left as well as a right hyperideal of $R$. That is, $x+y \subseteq I$ and $-x \in I$, for all $x, y \in I$ and $x \cdot y, y \cdot x \in I$, for all $x \in I$ and $y \in R$. Every hyperideal (whether left, right, two-sided) is a subhyperring of $R$ but the converse is not true in general.

Lemma 2.4. [14] A non-empty subset $A$ of a Krasner hyperring $R$ is a left (resp. right) hyperideal if and only if

(1) $a, b \in A$ implies $a-b \subseteq A$.

(2) $a \in A, r \in R$ imply $r \cdot a \in A$ (resp. $a \cdot r \in A)$.

Definition 2.5. [1] A partially ordered ring is a ring $(R,+, \cdot)$ together with a partial order $\leq$ in which $\leq$ is compatible with the addition and multiplication of $R$ in the following sense

(1) For all $a, b, x \in R, a \leq b$ implies that $a+x \leq b+x$,

(2) If $a, b, r \in R$ with $a \leq b$ and $0 \leq r$, then $a \cdot r \leq b \cdot r$ and $r \cdot a \leq r \cdot b$.

\section{Operations on hyperideals}

In this section, we deal with ordered Krasner hyperrings. Moreover, we study some aspects of hyperideals of ordered Krasner hyperrings.

Definition 3.1. A hypersructure $(R,+, \cdot, \leq)$ is called an ordered Krasner hyperring if the following conditions hold: 
(1) $(R,+, \cdot)$ is a Krasner hyperring.

(2) $(R, \leq)$ is a partially ordered set.

(3) For any $a, b, c \in R, a \leq b$ implies $a+c \leq b+c$, meaning that for any $x \in a+c$, there exists $y \in b+c$ such that $x \leq y$.

(4) For any $a, b, c \in R, a \leq b$ and $0 \leq c$ implies $a \cdot c \leq b \cdot c$ and $c \cdot a \leq c \cdot b$.

In what follows, we provide some examples of ordered Krasner hyperrings.

ExAMPLE 1. Every Krasner hyperring induces an ordered Krasner hyperring. Indeed: Let $(R,+, \cdot)$ be a Krasner hyperring. Define the order on $R$ by $\leq:=$ $\{(x, y): x=y\}$. Then $(R,+, \cdot, \leq)$ is an ordered Krasner hyperring.

Let $\left(R_{1},+_{1}, \cdot{ }_{1}, \leq_{1}\right)$ and $\left(R_{2},+_{2}, \cdot_{2}, \leq_{2}\right)$ be two ordered Krasner hyperrings. Then, the direct product of ordered Krasner hyperrings $R_{1}$ and $R_{2}$, is an ordered Krasner hyperring where for all $\left(s_{1}, s_{2}\right)$ and $\left(t_{1}, t_{2}\right)$ in $R_{1} \times R_{2}$ we define

(1) $\left(s_{1}, s_{2}\right)+\left(t_{1}, t_{2}\right)=\left\{(x, y): x \in s_{1}+{ }_{1} t_{1}, y \in s_{2}+{ }_{2} t_{2}\right\}$,

(2) $\left(s_{1}, s_{2}\right) \cdot\left(t_{1}, t_{2}\right)=\left(s_{1} \cdot t_{1}, s_{2} \cdot 2 t_{2}\right)$,

(3) $\left(s_{1}, s_{2}\right) \leq\left(t_{1}, t_{2}\right)$ if and only if $s_{1} \leq_{1} t_{1}$ and $s_{2} \leq_{2} t_{2}$.

In the following, we give another example in more detail.

EXAMPLE 2. A preorder on an arbitrary non-empty set $X$ is a binary relation on $X$ which is reflexive and transitive. An antisymmetric preorder is said to be an order. Let $\rho$ be a preorder relation on a Krasner hyperring $(R,+, \cdot)$. We say that $\rho$ is stable if for every $a, b, x \in R, a \rho b$ implies $a+x \rho b+x, x \cdot a \rho x \cdot b$ and $a \cdot x \rho b \cdot x$. Let $\rho$ be a stable preorder on a Krasner hyperring $R$. We construct an ordered Krasner hyperring $R / \rho=\left(R / \sim_{\rho}, \oplus, \odot, \leq\right)$. We define a binary relation $\sim_{\rho}$ on $R$ as follows: for every $a, b \in R, a \sim{ }_{\rho} b \Leftrightarrow a \rho b$ and $b \rho a$. Then, $\sim_{\rho}$ is a congruence relation on $R$. It can be shown easily that $\sim_{\rho}$ is an equivalence relation on $R$. Let $x, y, u, v \in R$ such that $x \sim_{\rho} y$ and $u \sim_{\rho} v$. Then $x \rho y, y \rho x, u \rho v$ and $v \rho u$. Since $\rho$ is a stable preorder on $R$, it follows that $x \cdot u \rho x \cdot v$ and $x \cdot v \rho y \cdot v$. Since $\rho$ is transitive, it follows that $x \cdot u \rho y \cdot v$. Similarly, we obtain $y \cdot v \rho x \cdot u$. Thus we have $x \cdot u \sim_{\rho} y \cdot v$. Similarly, we get $x+u \sim_{\rho} y+v$. Hence $\sim_{\rho}$ is a congruence relation on $R$. We write $[a]$ for the congruence class containing a specified element $a$. Now, let $R / \sim_{\rho}=\{[a]: a \in R\}$ be the set of equivalence classes. The congruence $\sim \rho$ determines a Krasner hyperring $\left(R / \sim_{\rho}, \oplus, \odot\right)$ with the hyperoperation $\oplus$ and the binary operation $\odot$ defined as follows:

$$
[a] \oplus[b]=\{[z]: z \in a+b\}
$$




$$
[a] \odot[b]=[a \cdot b] .
$$

The definition of a congruence ensures that $\oplus$ and $\odot$ are well-defined. We define an order relation $\leq$ on $R / \sim_{\rho}$ as follows: for any $a, b \in R,[a] \leq[b] \Leftrightarrow$ $a \rho b$. It is easy to see that $\left(R / \sim_{\rho}, \oplus, \odot, \leq\right)$ is an ordered Krasner hyperring.

Definition 3.2. Let $(R,+, \cdot, \leq)$ be an ordered Krasner hyperring. A nonempty subset $I$ of $R$ is called a hyperideal of $R$ if it satisfies the following conditions:

(1) $(I,+)$ is a canonical subhypergroup of $(R,+)$;

(2) $x \cdot y \in I$ and $y \cdot x \in I$ for all $x \in I$ and $y \in R$;

(3) When $x \in I$ and $y \in R$ such that $y \leq x$, imply that $y \in I$.

EXAMPLE 3 . Let $R=\{0, a, b, c\}$ be a set with the hyperaddition $\oplus$ and the multiplication $\odot$ defined as follows:

\begin{tabular}{|c|cccc|}
\hline$\oplus$ & 0 & $a$ & $b$ & $c$ \\
\hline 0 & 0 & $a$ & $b$ & $c$ \\
$a$ & $a$ & $\{0, b\}$ & $\{a, c\}$ & $b$ \\
$b$ & $b$ & $\{a, c\}$ & $\{0, b\}$ & $a$ \\
$c$ & $c$ & $b$ & $a$ & 0 \\
\hline
\end{tabular}

\begin{tabular}{|c|cccc|}
\hline$\odot$ & 0 & $a$ & $b$ & $c$ \\
\hline 0 & 0 & 0 & 0 & 0 \\
$a$ & 0 & $a$ & $b$ & $c$ \\
$b$ & 0 & $b$ & $b$ & 0 \\
$c$ & 0 & $c$ & 0 & $c$ \\
\hline
\end{tabular}

Then, $(R, \oplus, \odot)$ is a Krasner hyperring [2]. We have $(R, \oplus, \odot, \leq)$ is an ordered Krasner hyperring where the order relation $\leq$ is defined by:

$$
\leq:=\{(0,0),(a, a),(b, b),(c, c),(0, b),(c, a)\} .
$$

The covering relation and the figure of $R$ are given by:

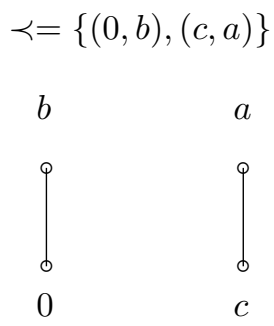

It is easy to see that $I_{1}=\{0\}, I_{2}=\{0, b\}, I_{3}=\{0, c\}, I_{4}=\{0, b, c\}$ and $I_{5}=\{0, a, b, c\}$ are hyperideals of $R$.

Lemma 3.3. Let $(R,+, \cdot, \leq)$ be an ordered Krasner hyperring. Then, 
(1) If $\left\{A_{k}: k \in \Lambda\right\}$ is a family of hyperideals of $R$, then $\bigcup_{k \in \Lambda} A_{k}$ is a hyperideal of $R$.

(2) If $\left\{A_{k}: k \in \Lambda\right\}$ is a family of hyperideals of $R$, then $\bigcap_{k \in \Lambda} A_{k}$ is a hyperideal of $R$.

Proof. (1): Since $0 \in \bigcup_{k \in \Lambda} A_{k}$, it follows that $\bigcup_{k \in \Lambda} A_{k} \neq \emptyset$. Let $a, b \in \bigcup_{k \in \Lambda} A_{k}$. Then $a, b \in A_{k}$ for some $k \in \Lambda$. Since $A_{k}$ is a hyperideal of $R$, we obtain $a-b \subseteq A_{k}$ for some $k \in \Lambda$. Thus $a-b \subseteq \bigcup_{k \in \Lambda} A_{k}$. Also we have $\left(\bigcup_{k \in \Lambda} A_{k}\right) \cdot R=$ $\bigcup_{k \in \Lambda} A_{k} \cdot R \subseteq \bigcup_{k \in \Lambda} A_{k}$ and $R \cdot\left(\bigcup_{k \in \Lambda} A_{k}\right)=\bigcup_{k \in \Lambda} R \cdot A_{k} \subseteq \bigcup_{k \in \Lambda} A_{k}$. So, for each $a \in \bigcup_{k \in \Lambda} A_{k}$ and $r \in R, a \cdot r \in \bigcup_{k \in \Lambda} A_{k}$. Similarly, $r \cdot a \in \bigcup_{k \in \Lambda} A_{k}$. Now, let $x \in \bigcup_{k \in \Lambda} A_{k}, y \in R$ and $y \leq x$. Then $x \in A_{k}$ for some $k \in \Lambda$. Since $A_{k}$ is a hyperideal of $R$, it follows that $y \in A_{k} \subseteq \bigcup_{k \in \Lambda} A_{k}$. Therefore, $\bigcup_{k \in \Lambda} A_{k}$ is a hyperideal of $R$, as desired.

(2): Since $0 \in \bigcap_{k \in \Lambda} A_{k}$, it follows that $\bigcap_{k \in \Lambda} A_{k} \neq \emptyset$. Let $a, b \in \bigcap_{k \in \Lambda} A_{k}$ and $r \in R$. Then $a, b \in A_{k}$ for each $k \in \Lambda$. By assumption, we obtain $a-b \subseteq A_{k}$ for each $k \in \Lambda$. Thus $a-b \subseteq \bigcap_{k \in \Lambda} A_{k}$. Similarly $r \cdot a, a \cdot r \in \bigcap_{k \in \Lambda} A_{k}$. Now, let $x \in \bigcap_{k \in \Lambda} A_{k}$ and $y \in R$ such that $y \leq x$. Then for every $k \in \Lambda, y \in A_{k}$. Hence $y \in \bigcap_{k \in \Lambda} A_{k}$. Therefore, $\bigcap_{k \in \Lambda} A_{k}$ is a hyperideal of $R$.

We say that a preorder relation is a relation which satisfies conditions reflexivity and transitivity. We continue this section with the following theorem.

Theorem 3.4. Let $(R,+, \cdot, \leq)$ be a preordered Krasner hyperring and $\rho$ be a strongly regular relation on $R$. Then, $(R / \rho, \oplus, \odot, \preceq)$ is a preordered ring with respect to the following hyperoperations on the quotient set $R / \rho$ :

$$
\begin{gathered}
\bar{a} \oplus \bar{b}=\{\bar{c} \mid c \in a+b\}, \\
\bar{a} \odot \bar{b}=\overline{a \cdot b},
\end{gathered}
$$

where for all $\bar{a}, \bar{b} \in R / \rho$ a preorder relation $\preceq$ is defined by:

$$
\bar{a} \preceq \bar{b} \Leftrightarrow \forall a_{1} \in \bar{a} \exists b_{1} \in \bar{b} \text { such that } a_{1} \leq b_{1} .
$$

Proof. Since $\rho$ is a strongly regular relation on $R$, it follows that $(R / \rho, \oplus, \odot)$ is a ring. First, we show that the binary relation $\preceq$ is a preorder relation 
on $R / \rho$. Since $\leq$ is reflexive, it follows that $(a, a) \in \leq$. So, $\bar{a} \preceq \bar{a}$ for every $\bar{a} \in R / \rho$. Thus $\preceq$ is reflexive. Now, let $\bar{a} \preceq \bar{b}$ and $\bar{b} \preceq \bar{c}$. Then for every $a_{1} \in \bar{a}$ there exists $b_{1} \in \bar{b}$ such that $a_{1} \leq b_{1}$. Since $b_{1} \in \bar{b} \preceq \bar{c}$, there exists $c_{1} \in \bar{c}$ such that $b_{1} \leq c_{1}$. So, $\bar{a} \preceq \bar{c}$. Hence $\preceq$ is transitive. Therefore, the binary relation $\preceq$ is a preorder relation on $R / \rho$. Now, let $\bar{a}, \bar{b}, \bar{x} \in R / \rho$ such that $\bar{a} \preceq \bar{b}$. If $\bar{u}=\bar{x} \oplus \bar{a}$, then for every $u_{1} \in \bar{u}$ there exist $x_{1} \in \bar{x}$ and $a_{1} \in \bar{a}$ such that $u_{1} \in x_{1}+a_{1}$. Since $a_{1} \in \bar{a} \preceq \bar{b}$, there exists $b_{1} \in \bar{b}$ such that $a_{1} \leq b_{1}$. Hence $x_{1}+a_{1} \leq x_{1}+b_{1}$. Thus there exists $v_{1} \in x_{1}+b_{1}$ such that $u_{1} \leq v_{1}$. Hence $\bar{u}=\overline{u_{1}} \preceq \overline{v_{1}}=\bar{x} \oplus \bar{b}$. So, we have $\bar{x} \oplus \bar{a} \preceq \bar{x} \oplus \bar{b}$. If $\bar{s}=\bar{x} \odot \bar{a}$, then for every $s_{1} \in \bar{s}$ there exist $x_{1} \in \bar{x}$ and $a_{1} \in \bar{a}$ such that $s_{1}=x_{1} \cdot a_{1}$. Since $a_{1} \in \bar{a} \preceq \bar{b}$, there exists $b_{1} \in \bar{b}$ such that $a_{1} \leq b_{1}$. Hence $x_{1} \cdot a_{1} \leq x_{1} \cdot b_{1}$. Thus for $t_{1}=x_{1} \cdot b_{1}$, we have $s_{1} \leq t_{1}$. Hence $\bar{s}=\overline{s_{1}} \preceq \overline{t_{1}}=\bar{x} \odot \bar{b}$. Therefore, $(R / \rho, \oplus, \odot, \preceq)$ is a preordered ring.

Definition 3.5. Let $\left(R_{1},+_{1}, \cdot_{1}, \leq_{1}\right)$ and $\left(R_{2},+_{2}, \cdot_{2}, \leq_{2}\right)$ be two ordered Krasner hyperrings. The map $\varphi: R_{1} \rightarrow R_{2}$ is called a homomorphism if for all $a, b \in R_{1}$, the followinging conditions hold:

(1) $\varphi\left(a+{ }_{1} b\right) \subseteq \varphi(a)+{ }_{2} \varphi(b)$,

(2) $\varphi\left(a \cdot{ }_{1} b\right)=\varphi(a) \cdot 2 \varphi(b)$,

(3) $a \leq_{1} b$ implies that $\varphi(a) \leq_{2} \varphi(b)$.

Also $\varphi$ is called a good (strong) homomorphism if in the previous condition (1), the equality is valid. An isomorphism from $\left(R_{1},+_{1}, \cdot_{1}, \leq_{1}\right)$ into $\left(R_{2},+_{2}, \cdot_{2}, \leq_{2}\right)$ is a bijective good homomophism from $\left(R_{1},+_{1}, \cdot_{1}, \leq_{1}\right)$ onto $\left(R_{2},+_{2}, \cdot_{2}, \leq_{2}\right)$. The kernel of $\varphi, \operatorname{ker} \varphi$, is defined by $\operatorname{ker} \varphi=\left\{x \in R_{1} \mid \varphi(x)=\right.$ $\left.0_{2}\right\}$, where $0_{2}$ is the zero of $R_{2}$.

Theorem 3.6. Let $\varphi$ be a homomorphism from an ordered Krasner hyperring $(R,+, \cdot, \leq)$ into an ordered Krasner hyperring $(T, \oplus, \odot, \preceq)$. If I is a hyperideal of $T$, then $\varphi^{-1}(I)=\{a \in R: \varphi(a) \in I\}$ is a hyperideal of $R$ containing ker $\varphi$.

Proof. Since $0 \in \varphi^{-1}(I)$, it follows that $\varphi^{-1}(I) \neq \emptyset$. Let $x \in R$. Since $\varphi$ is a homomorphism and $0 \in x-x$, we have $0=\varphi(0) \in \varphi(x-x) \subseteq \varphi(x) \oplus \varphi(-x)$. So $0 \in \varphi(x) \oplus \varphi(-x)$. Thus, $\varphi(-x)$ is the inverse of $\varphi(x)$ in the canonical hypergroup $(T, \oplus)$. Since $0 \in \varphi(x) \oplus \varphi(-x)$, it follows that $\varphi(-x)=-\varphi(x)$. Now, let $a_{1}, a_{2} \in \varphi^{-1}(I)$. Then $\varphi\left(a_{1}\right), \varphi\left(a_{2}\right) \in I$. Since $I$ is a hyperideal of $T$, we have $\varphi\left(a_{1}-a_{2}\right) \subseteq \varphi\left(a_{1}\right) \ominus \varphi\left(a_{2}\right) \subseteq I$. Hence $a_{1}-a_{2} \subseteq \varphi^{-1}(I)$. Let $x \in R$ and $a \in \varphi^{-1}(I)$. Then $\varphi(a) \in I$. Since $\varphi$ is a homomorphism, it follows that $\varphi(x \cdot a)=\varphi(x) \odot \varphi(a) \in I$. Thus $x \cdot a \in \varphi^{-1}(I)$. Similarly, $a \cdot x \in \varphi^{-1}(I)$. Now, let $a \in \varphi^{-1}(I)$ and $b \in R$ such that $b \leq a$. Then $\varphi(a) \in I$. Since $b \leq a$ and $\varphi$ is a homomorphism, we have $\varphi(b) \preceq \varphi(a)$. Since $I$ is a hyperideal of 
$T$, it follows that $\varphi(b) \in I$. So $b \in \varphi^{-1}(I)$. This proves that $\varphi^{-1}(I)$ is a hyperideal of $R$, as desired. Moreover, if $x \in \operatorname{ker} \varphi$, then $\varphi(x)=0 \in I$. Hence $x \in \varphi^{-1}(I)$. Therefore, $\operatorname{ker} \varphi \subseteq \varphi^{-1}(I)$.

We continue this section with the following definition.

Definition 3.7. Let $I$ and $J$ be two hyperideals of an ordered Krasner hyperring $(R,+, \cdot, \leq)$. The hyperideal quotient is $(I: J)=\{x \in R: x \cdot J \subseteq I\}$. The hyperideal quotient $(0: J)$ is called the annihilator of $J$ and denoted by $\operatorname{Ann}(J)$. The set $\operatorname{Ann}_{l}(x)=\{a \in R: a \cdot x=0\}$ is called the left annihilator of $x$ in $R$. Similarly, the set $\operatorname{Ann}_{r}(x)=\{a \in R: x \cdot a=0\}$ is called the right annihilator of $x$ in $R$. In a commutative ordered Krasner hyperring $R$, we have $A n n_{l}(x)=A n n_{r}(x)$. In this case, we denote it by $A n n(x)$.

Lemma 3.8. In Definition 3.7, Ann $(x)$ is a hyperideal of $R$.

Proof. Since $0 \in A n n(x)$, it follows that $\operatorname{Ann}(x) \neq \emptyset$. Let $a, b \in A n n(x)$. Then $a \cdot x=0$ and $b \cdot x=0$. So, we have $(a+b) \cdot x=a \cdot x+b \cdot x=0+0=0$. Thus $c \cdot x=0$ fo all $c \in a+b$. Hence $a+b \subseteq \operatorname{Ann}(x)$. Also, we have $(-a) \cdot x=-(a \cdot x)=-0=0$. So, $-a \in \operatorname{Ann}(x)$. Now, let $a \in \operatorname{Ann}(x)$ and $r \in R$. Since $a \cdot x=0$, it follows that $(r \cdot a) \cdot x=r \cdot(a \cdot x)=r \cdot 0=0$. So, we have $r \cdot a \in \operatorname{Ann}(x)$. Let $a \in \operatorname{Ann}(x), b \in R$ and $b \leq a$. Then, we have $b \cdot x \leq a \cdot x$. Since $a \cdot x=0$ and $\{0\}$ is a hyperideal of $R$, we obtain $b \cdot x=0$. So, $b \in \operatorname{Ann}(x)$. Therfore, $\operatorname{Ann}(x)$ is a hyperideal of $R$, as desired.

Example 4. In Example 3, Ann $(0)=\{0, a, b, c\}, \operatorname{Ann}(a)=\{0\}, \operatorname{Ann}(b)=$ $\{0, c\}$ and $\operatorname{Ann}(c)=\{0, b\}$ which are hyperideals of $R$.

Theorem 3.9. In Definition 3.7, $(I: J)$ is a hyperideal of $R$.

Proof. This proof is straightforward.

Definition 3.10. Let $(R,+, \cdot, \leq)$ be an ordered Krasner hyperring (resp. Krasner hyperring). $R$ is said to be a reduced ordered Krasner hyperring (resp. reduced Krasner hyperring) if it has no nilpotent elements, i.e., if $a^{n}=0$ for $a \in R$ and a natural number $n$, then $a=0$. In a reduced ordered Krasner hyperring $R$, If $a \cdot b=0$ for all $a, b \in R$, then $b \cdot a=0$. So, we have $A n n_{l}(x)=A n n_{r}(x)$. In this case, we denote it by $A n n(x)$.

REMARK 1. In Definition 3.7, we can replace the commutative ordered Krasner hyperring with the reduced ordered Krasner hyperring. 


\section{Properties of interior hyperideals in ordered Krasner hyperrings}

In this section, we introduce the notion of interior hyperideals in ordered Krasner hyperrings and investigate some related results. We provide conditions for an interior hyperideal to be a hyperideal. In particular, we prove that the concepts of interior hyperideals and hyperideals coincide in the case of regular (resp. intra-regular) ordered Krasner hyperrings.

Definition 4.1. A non-empty subset $A$ of an ordered Krasner hyperring $(R,+, \cdot, \leq)$ is called an interior hyperideal of $R$ if the following conditions hold:

(1) $(A,+)$ is a canonical subhypergroup of $(R,+)$ and $A \cdot A \subseteq A$;

(2) $R \cdot A \cdot R \subseteq A$;

(3) When $x \in A$ and $y \in R$ such that $y \leq x$, imply that $y \in A$.

ExAmPLE 5 . Let $R=\{a, b, c, d, e, f, g, h\}$ be a set with the hyperaddition $\oplus$ and the multiplication $\odot$ defined as follows:

\begin{tabular}{|c|cccccccc|}
\hline$\oplus$ & $a$ & $b$ & $c$ & $d$ & $e$ & $f$ & $g$ & $h$ \\
\hline$a$ & $a$ & $b$ & $c$ & $d$ & $e$ & $f$ & $g$ & $h$ \\
$b$ & $b$ & $b$ & $\{a, b, c, d\}$ & $b$ & $f$ & $f$ & $\{e, f, g, h\}$ & $f$ \\
$c$ & $c$ & $\{a, b, c, d\}$ & $c$ & $c$ & $g$ & $\{e, f, g, h\}$ & $g$ & $g$ \\
$d$ & $d$ & $b$ & $c$ & $a$ & $h$ & $f$ & $g$ & $e$ \\
$e$ & $e$ & $f$ & $g$ & $h$ & $\{a, e\}$ & $\{b, f\}$ & $\{c, g\}$ & $\{d, h\}$ \\
$f$ & $f$ & $f$ & $\{e, f, g, h\}$ & $f$ & $\{b, f\}$ & $\{b, f\}$ & $R$ & $\{b, f\}$ \\
$g$ & $g$ & $\{e, f, g, h\}$ & $g$ & $g$ & $\{c, g\}$ & $R$ & $\{c, g\}$ & $\{c, g\}$ \\
$h$ & $h$ & $f$ & $g$ & $e$ & $\{d, h\}$ & $\{b, f\}$ & $\{c, g\}$ & $\{a, e\}$ \\
\hline
\end{tabular}

and

\begin{tabular}{|l|llllllll|}
$\odot$ & $a$ & $b$ & $c$ & $d$ & $e$ & $f$ & $g$ & $h$ \\
\hline$a$ & $a$ & $a$ & $a$ & $a$ & $a$ & $a$ & $a$ & $a$ \\
$b$ & $a$ & $b$ & $c$ & $d$ & $a$ & $b$ & $c$ & $d$ \\
$c$ & $a$ & $c$ & $b$ & $d$ & $a$ & $c$ & $b$ & $d$ \\
$d$ & $a$ & $a$ & $a$ & $a$ & $a$ & $a$ & $a$ & $a$ \\
$e$ & $a$ & $a$ & $a$ & $a$ & $e$ & $e$ & $e$ & $e$ \\
$f$ & $a$ & $b$ & $c$ & $d$ & $e$ & $f$ & $g$ & $h$ \\
$g$ & $a$ & $c$ & $b$ & $d$ & $e$ & $g$ & $f$ & $h$ \\
$h$ & $a$ & $a$ & $a$ & $a$ & $e$ & $e$ & $e$ & $e$ \\
\hline
\end{tabular}


Then, $(R, \oplus, \odot)$ is a Krasner hyperring. We have $(R, \oplus, \odot, \leq)$ is an ordered Krasner hyperring where the order relation $\leq$ is defined by:

$$
\begin{aligned}
\leq \quad:= & \{(a, a),(b, b),(c, c),(d, d),(e, e),(f, f),(g, g), \\
& (h, h),(a, e),(b, f),(c, g),(d, h)\} .
\end{aligned}
$$

The covering relation and the figure of $R$ are given by:

$$
\prec \quad=\{(a, e),(b, f),(c, g),(d, h)\} .
$$

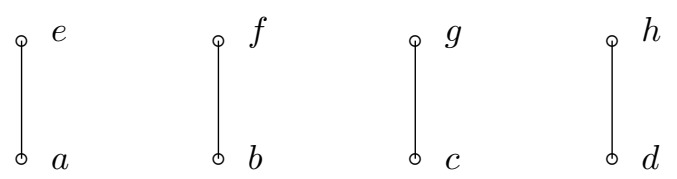

It is easy to see that $\{a\},\{a, d\},\{a, e\}\{a, b, c, d\},\{a, d, e, h\}$ and $R$ are interior hyperideals of $R$.

Obviously, every hyperideal of an ordered Krasner hyperring $R$ is an interior hyperideal, but the converse is not true in general, that is, an interior hyperideal may not be a hyperideal of $R$.

ExAmple 6 . Let $R$ be the set $\left\{\left(\begin{array}{ll}a & 0 \\ b & c\end{array}\right): a, b, c \in \mathbb{Z}\right\}$. We define the binary hyperoperation $\oplus$ as: $A \oplus B=\{A+B\}$. Consider the operation $\odot$ as usual matrix multiplication. Then, $(R, \oplus, \odot)$ is a Krasner hyperring. Moreover, $(R, \oplus, \odot, \leq)$ is an ordered Krasner hyperring, where $A=\left(a_{i j}\right) \leq B=\left(b_{i j}\right) \Leftrightarrow$ $a_{i j}=b_{i j}$ for all $1 \leqslant i, j \leqslant 2$. Let $A=\left\{\left(\begin{array}{ll}a & 0 \\ b & 0\end{array}\right): a, b \in \mathbb{Z}\right\}$. It is easy to check that $A$ is an interior hyperideal of $R$. Since $\left(\begin{array}{ll}1 & 0 \\ 0 & 0\end{array}\right) \odot\left(\begin{array}{ll}0 & 1 \\ 0 & 0\end{array}\right)=\left(\begin{array}{ll}0 & 1 \\ 0 & 0\end{array}\right) \notin A$, it follows that $A$ is not a right hyperideal of $R$. Thus $A$ is not a hyperideal of $R$.

Lemma 4.2. Let $(R,+, \cdot, \leq)$ be an ordered Krasner hyperring. If $A_{k}$ is an interior hyperideal of $R$ for all $k \in \Lambda$, then $\bigcap_{k \in \Lambda} A_{k}$ is an interior hyperideal of $R$.

Proof. Let $\left\{A_{k}: k \in \Lambda\right\}$ be a family of interior hyperideals of $R$ and $A=$ $\bigcap_{k \in \Lambda} A_{k}$. Since $0 \in \bigcap_{k \in \Lambda} A_{k}$, it follows that $\bigcap_{k \in \Lambda} A_{k} \neq \emptyset$. It is easy to check 
that $(A,+)$ is a canonical subhypergroup of $(R,+)$ and $A \cdot A \subseteq A$. Now, let $x \in R \cdot A \cdot R$. Then $x=r_{1} \cdot a \cdot r_{2}$ for some $r_{1}, r_{2} \in R$ and $a \in A$. Since each $A_{k}$ is an interior hyperideal of $R$, it follows that $x \in R \cdot A_{k} \cdot R \subseteq A_{k}$ for all $k \in \Lambda$. Thus $x \in A_{k}$ for all $k \in \Lambda$. Hence $x \in \bigcap_{k \in \Lambda} A_{k}=A$. Since $x$ was chosen arbitrarily, it follows that $R \cdot A \cdot R \subseteq A$. If $x \in A$ and $y \in R$ such that $y \leq x$, then $x \in A_{k}$ for all $k \in \Lambda$. Since each $A_{k}$ is an interior hyperideal of $R$, it follows that $y \in A_{k}$ for all $k \in \Lambda$. Thus we have $y \in \bigcap_{k \in \Lambda} A_{k}=A$. Therefore, $A$ is an interior hyperideal of $R$.

Theorem 4.3. Let $(R,+, \cdot, \leq)$ and $(T, \oplus, \odot, \preceq)$ be two ordered Krasner hyperrings. If $\varphi: R \rightarrow T$ is a homomorphism and $A$ is an interior hyperideal of $T$, then $\varphi^{-1}(A)=\{r \in R: \varphi(r) \in A\}$ is an interior hyperideal of $R$.

Proof. Since $0 \in \varphi^{-1}(A)$, it follows that $\varphi^{-1}(A) \neq \emptyset$. Now, let $r_{1}, r_{2} \in$ $\varphi^{-1}(A)$. Then $\varphi\left(r_{1}\right), \varphi\left(r_{2}\right) \in A$. Since $A$ is an interior hyperideal of $T$, we have $\varphi\left(r_{1}+r_{2}\right) \subseteq \varphi\left(r_{1}\right) \oplus \varphi\left(r_{2}\right) \subseteq A$ and $\varphi\left(r_{1} \cdot r_{2}\right)=\varphi\left(r_{1}\right) \odot \varphi\left(r_{2}\right) \in A$. Thus $r_{1}+r_{2} \subseteq \varphi^{-1}(A)$ and $r_{1} \cdot r_{2} \in \varphi^{-1}(A)$. obviously, other properties of a hyperring hold for $\varphi^{-1}(A)$, since $\varphi^{-1}(A)$ is a subset of $R$. Let $r_{1}, r_{2} \in R$ and $a \in \varphi^{-1}(A)$. Then $\varphi\left(r_{1}\right), \varphi\left(r_{2}\right) \in T$ and $\varphi(a) \in A$. Since $A$ is an interior hyperideal of $T$, it follows that $\varphi\left(r_{1} \cdot a \cdot r_{2}\right)=\varphi\left(r_{1}\right) \odot \varphi(a) \odot \varphi\left(r_{2}\right) \in A$. So, we have $r_{1} \cdot a \cdot r_{2} \in \varphi^{-1}(A)$. Hence $R \cdot \varphi^{-1}(A) \cdot R \subseteq \varphi^{-1}(A)$. Now, let $a \in \varphi^{-1}(A)$ and $r \in R$ such that $r \leq a$. Since $\varphi$ is a homomorphism, it follows that $\varphi(r) \preceq \varphi(a)$. Since $\varphi(a) \in A$ and $A$ is an interior hyperideal of $T$, we obtain $\varphi(r) \in A$. So, we have $r \in \varphi^{-1}(A)$. Therefore, $\varphi^{-1}(A)$ is an interior hyperideal of $R$.

In the following, we provide conditions for an interior hyperideal to be a hyperideal.

Theorem 4.4. Let $(R,+, \cdot, \leq)$ be an ordered Krasner hyperring. Then,

(1) If $R$ is a regular ordered Krasner hyperring, then every interior hyperideal of $R$ is a hyperideal of $R$.

(2) If $R$ is an intra-regular ordered Krasner hyperring, then every interior hyperideal of $R$ is a hyperideal of $R$.

Proof. (1): Let $A$ be an interior hyperideal of $R$ and $a \in A$. Since $R$ is regular, there exists $x \in R$ such that $a \leq a \cdot x \cdot a$. Now, let $r \in R$. Since $A$ is an interior hyperideal of $R$, it follows that $a \cdot r \leq(a \cdot x \cdot a) \cdot r=(a \cdot x) \cdot a \cdot r \in A \cdot A \subseteq A$. Since $r$ was chosen arbitrarily, we have $A \cdot R \subseteq A$. Hence $A$ is a right hyperideal of $R$. Similarly, we can prove that $A$ is a left hyperideal of $R$. Therefore, $A$ is a hyperideal of $R$. 
(2): Let $A$ be an interior hyperideal of $R$. Let $r \in R$ and $a \in A$. Since $R$ is intra-regular, there exist $x, y \in R$ such that $a \leq x \cdot a^{2} \cdot y$. Since $A$ is an interior hyperideal of $R$, it follows that $a \cdot r \leq\left(x \cdot a^{2} \cdot y\right) \cdot r=(x \cdot a) \cdot a \cdot(y \cdot r) \in A$. Since $r$ was chosen arbitrarily, we have $A \cdot R \subseteq A$. Hence $A$ is a right hyperideal of $R$. Similarly, we can prove that $A$ is a left hyperideal of $R$. Therefore, $A$ is a hyperideal of $R$.

As a consequence we obtain the following corollary.

Corollary 4.5. Let $(R,+, \cdot, \leq)$ be a regular (resp. intra-regular) ordered Krasner hyperring. Then, the following assertions are equivalent:

(1) $A$ is a hyperideal of $R$.

(2) $A$ is an interior hyperideal of $R$.

The concepts of regular (resp. intra-regular) ordered Krasner hyperrings generalize the corresponding concepts of regular (resp. intra-regular) Krasner hyperrings as each regular (resp. intra-regular) Krasner hyperring endowed with the order $\leq:=\{(a, b): a=b\}$ is a regular (resp. intra-regular) ordered Krasner hyperring. Let $(R,+, \cdot, \leq)$ be an ordered Krasner hyperring and $\emptyset \neq$ $A \subseteq R$. Then, $(A]$ is the subset of $R$ defined as follows: $(A]=\{x \in R: x \leq a$ for some $a \in A\}$. Let $A, B \subseteq R$. Then $A \subseteq(A],(A](B] \subseteq(A B]$ and $((A]]=$ $(A]$. An element $a$ of an ordered Krasner hyperring $(R,+, \cdot, \leq)$ is said to be regular if there exists an element $x \in R$ such that $a \leq(a \cdot x) \cdot a$, i.e., $a \in(a R a]$ for all $a \in R$ or $A \subseteq(A R A]$ for all $A \subseteq R$. An ordered Krasner hyperring $(R,+, \cdot, \leq)$ is said to be regular if every element of $R$ is regular. An element $a$ of an ordered Krasner hyperring $(R,+, \cdot, \leq)$ is said to be intra-regular if there exist $x, y \in R$ such that $a \leq x \cdot a^{2} \cdot y$, i.e., $a \in\left(R a^{2} R\right]$ for all $a \in R$ or $A \subseteq\left(R A^{2} R\right]$ for all $A \subseteq R$. An ordered Krasner hyperring $(R,+, \cdot, \leq)$ is said to be intra-regular if every element of $R$ is intra-regular. An ordered Krasner hyperring $(R,+, \cdot, \leq)$ is called left (resp. right) regular if for every $a \in R$ there exists an element $x \in R$ such that $a \leq x \cdot a^{2}$ (resp. $a \leq a^{2} \cdot x$ ). An ordered Krasner hyperring $R$ is called left (resp. right) regular if all elements of $R$ are left (resp. right) regular, i.e., $a \in\left(R a^{2}\right]$, (resp. $\left.a \in\left(a^{2} R\right]\right) \forall a \in R$. or $A \subseteq\left(R A^{2}\right]$, (resp. $\left.A \subseteq\left(A^{2} R\right]\right) \forall A \subseteq R$. An ordered Krasner hyperring is called completely regular if it is regular, left regular and right regular.

Asokkumar [3] studied the idempotent elements of Krasner hyperrings.

Definition 4.6. Let $(R,+, \cdot, \leq)$ be an ordered Krasner hyperring (resp. Krasner hyperring). An element $x$ of $R$ is said to be idempotent if $\{x\}=x \cdot x=x^{2}$. An ordered Krasner hyperring (resp. Krasner hyperring) is called idempotent if every element $x$ of $R$ is an idempotent. An ordered Krasner hyperring 
(resp. Krasner hyperring) is called a Boolean ordered Krasner hyperring (resp. Boolean Krasner hyperring) if every element $x$ of $R$ is an idempotent.

The following theorem were motivated by the Corollary 4.5 .

Theorem 4.7. Let $(R,+, \cdot, \leq)$ be an ordered Krasner hyperring. Then, the following assertions hold:

(1) If $R$ is a Boolean ordered Krasner hyperring, then every interior hyperideal of $R$ is a hyperideal of $R$.

(2) If $R$ is left (resp. right) regular, then every interior hyperideal of $R$ is a hyperideal of $R$.

Proof. (1): Let $R$ be a Boolean ordered Krasner hyperring and $x \in R$. Then $\{x\}=x^{2}=x \cdot x=x^{2} \cdot x^{2}=x \cdot x^{2} \cdot x$. Since $\leq$ is reflexive, it follows that $(x, x) \in \leq$. So, $\left(x, x \cdot x^{2} \cdot x\right) \in \leq$. Thus $x \leq x \cdot x^{2} \cdot x$ for every $x \in R$. Hence $R$ is intra-regular. Hence by Corollary 4.5, every interior hyperideal of $R$ is a hyperideal of $R$.

(2): Let $R$ be a left regular ordered Krasner hyperring and $x \in R$. Then there exists an element $y \in R$ such that $x \leq y \cdot x^{2} \leq y \cdot\left(y \cdot x^{2}\right) \cdot x \in R x^{2} R$. So, $R$ is intra-regular. Hence by Corollary 4.5 , every interior hyperideal of $R$ is a hyperideal of $R$. Similarly, we can prove that in a right regular ordered Krasner hyperring $R$, every interior hyperideal of $R$ is a hyperideal of $R$.

\section{Main results}

The concepts of hyperideals, prime hyperideals and semiprime hyperideals of ordered Krasner hyperrings generalize the corresponding concepts of Krasner hyperrings. Let $(R,+, \cdot, \leq)$ be an ordered Krasner hyperring. A hyperideal $A$ of $R$ is called a prime hyperideal of $R$ if for any hyperideals $A_{1}, A_{2}$ of $R$ such that $A_{1} \cdot A_{2} \subseteq A$, we have $A_{1} \subseteq A$ or $A_{2} \subseteq A$. Note that if a hyperideal $A$ of $R$ is prime, then $A \neq R$. A hyperideal $A$ of $R$ is called a semiprime hyperideal of $R$ if for any hyperideal $B$ of $R$ such that $B^{2} \subseteq A$, we have $B \subseteq A$. Note that every prime hyperideal of $R$ is a semiprime hyperideal of $R$, but the converse is not true in general, that is, a semiprime hyperideal may not be a prime hyperideal of $R$.

ExAmple 7. (1) In Example 5, $\{a, b, c, d\}$ and $\{a, d, e, h\}$ are prime hyperideals of $R$, but $\{a\},\{a, d\}$ and $\{a, e\}$ are not prime hyperideals of $R$.

(2) In Example 3, $\{0\}$ is not a prime hyperideal of $R$. Indeed, $\{0, b\} \odot\{0, c\}=$ $\{0\}$, but $\{0, b\} \nsubseteq\{0\}$ and $\{0, c\} \nsubseteq\{0\}$. 
(3) In Example 3, \{0\} is a semiprime hyperideal of $R$, but is not a prime hyperideal of $R$.

(4) In Example $5,\{a, d\}$ is a semiprime hyperideal of $R$, but is not a prime hyperideal of $R$. Indeed, $\{a, b, c, d\} \odot\{a, d, e, h\}=\{a, d\}$, but $\{a, b, c, d\} \nsubseteq\{a, d\}$ and $\{a, d, e, h\} \nsubseteq\{a, d\}$.

Theorem 5.1. Let $(R,+, \cdot, \leq)$ be an ordered Krasner hyperring. Then, the following statements are equivalent:

(1) $R$ is intra-regular.

(2) Every interior hyperideal of $R$ is semiprime.

(3) Every hyperideal of $R$ is semiprime.

Proof. (1) $\Rightarrow(2)$ : Assume that (1) holds. Let $A$ be an interior hyperideal of $R$ and $B \subseteq R$ such that $B^{2} \subseteq A$. Since $R$ is intra-regular, we obtain $B \subseteq\left(R B^{2} R\right] \subseteq(R A R] \subseteq(A]=A$. So, $A$ is semiprime.

$(2) \Rightarrow(3)$ : This proof is straightforward.

$(3) \Rightarrow(1)$ : Assume that (3) holds. Let $A \subseteq R$. It is easy to see that $\left(R A^{2} R\right]$ is a hyperideal of $R$. By assumption, $\left(R A^{2} R\right]$ is semiprime. Since $A^{4} \subseteq\left(R A^{2} R\right]$, we have $A \subseteq\left(R A^{2} R\right]$. So, $R$ is intra-regular.

Definition 5.2. Let $(R,+, \cdot, \leq)$ be an ordered Krasner hyperring. A subhyperring $A$ of $R$ is called a bi-hyperideal of $R$ if $A \cdot R \cdot A \subseteq A$ and $(A] \subseteq A$. For every left hyperideal, right hyperideal, hyperideal and bi-hyperideal $A$ of $R$, we have $(A]=A$. A non-zero bi-hyperideal $A$ of $R$ is said to be a minimal bi-hyperideal if $A$ does not properly contain any non-zero bi-hyperideal contained in $A$.

Theorem 5.3. Let $(R,+, \cdot, \leq)$ and $(T, \oplus, \odot, \preceq)$ be two ordered Krasner hyperrings. If $\varphi: R \rightarrow T$ is a homomorphism and $A$ is a bi-hyperideal of $T$, then $\varphi^{-1}(A)=\{r \in R: \varphi(r) \in A\}$ is a bi-hyperideal of $R$.

Proof. Since $0 \in \varphi^{-1}(A)$, it follows that $\varphi^{-1}(A) \neq \emptyset$. Now, let $r_{1}, r_{2} \in$ $\varphi^{-1}(A)$. Then $\varphi\left(r_{1}\right), \varphi\left(r_{2}\right) \in A$. Since $A$ is a bi-hyperideal of $T$, we have $\varphi\left(r_{1}+r_{2}\right) \subseteq \varphi\left(r_{1}\right) \oplus \varphi\left(r_{2}\right) \subseteq A$ and $\varphi\left(r_{1} \cdot r_{2}\right)=\varphi\left(r_{1}\right) \odot \varphi\left(r_{2}\right) \in A$. Thus $r_{1}+r_{2} \subseteq \varphi^{-1}(A)$ and $r_{1} \cdot r_{2} \in \varphi^{-1}(A)$. obviously, other properties of a hyperring hold for $\varphi^{-1}(A)$, since $\varphi^{-1}(A)$ is a subset of $R$. Let $a_{1}, a_{2} \in \varphi^{-1}(A)$ and $x \in R$. Then $\varphi\left(a_{1}\right), \varphi\left(a_{2}\right) \in A$ and $\varphi(x) \in T$. Since $A$ is a bi-hyperideal of $T$, it follows that $\varphi\left(a_{1} \cdot x \cdot a_{2}\right)=\varphi\left(a_{1}\right) \odot \varphi(x) \odot \varphi\left(a_{2}\right) \in A$. So, we have $a_{1} \cdot x \cdot a_{2} \in \varphi^{-1}(A)$. Hence $\varphi^{-1}(A) \cdot R \cdot \varphi^{-1}(A) \subseteq \varphi^{-1}(A)$. Now, let $a \in \varphi^{-1}(A)$ and $x \in R$ such that $x \leq a$. Since $\varphi$ is a homomorphism, it follows that $\varphi(x) \preceq \varphi(a)$. Since $\varphi(a) \in A$ and $A$ is a bi-hyperideal of $T$, we obtain 
$\varphi(x) \in A$. Thus we have $x \in \varphi^{-1}(A)$. Therefore, $\varphi^{-1}(A)$ is a bi-hyperideal of $R$.

Theorem 5.4. Let $(R,+, \cdot, \leq)$ be an ordered Krasner hyperring, having a non-zero proper bi-hyperideal. Then, every non-zero proper bi-hyperideal of $R$ is minimal if and only if the intersection of any two distinct non-zero proper bi-hyperideals is $\{0\}$.

Proof. Assume that every non-zero proper bi-hyperideal of $R$ is minimal. Let $A_{1}$ and $A_{2}$ be two distinct non-zero proper bi-hyperideals of $R$ and $A_{1} \cap A_{2} \neq$ $\{0\}$. It is easy to see that $A_{1} \cap A_{2}$ is a bi-hyperideal of $R$. By hypothesis, $A_{1}$ and $A_{2}$ are minimal. Since $\{0\} \neq A_{1} \cap A_{2} \subseteq A_{1}$ and $\{0\} \neq A_{1} \cap A_{2} \subseteq A_{1}$, we obtain $A_{1}=A_{2}$; a contradiction. So $A_{1} \cap A_{2}=\{0\}$, as desired.

Theorem 5.5. Let $(R,+, \cdot, \leq)$ be an ordered Krasner hyperring. Then,

(1) If $R$ is left regular, then the left hyperideals of $R$ are semiprime.

(2) If $R$ is completely regular, then every bi-hyperideal of $R$ is semiprime.

Proof. (1): Let $A$ be a left hyperideal of $R$ and $T \subseteq R$ such that $T^{2} \subseteq A$. Since $R$ is left regular, we have $T \subseteq\left(R T^{2}\right] \subseteq(R A] \subseteq(A]=A$. So, $A$ is semiprime.

(2): Let $A$ be a bi-hyperideal of $R$. Let $T$ be a bi-hyperideal of $R$ such that $T^{2} \subseteq A$. Since $R$ is completely regular, we have

$$
\begin{aligned}
T \subseteq(T R T] & \subseteq\left(\left(T^{2} R\right] R\left(R T^{2}\right]\right]=\left(\left(T^{2} R\right](R]\left(R T^{2}\right]\right) \\
& \subseteq\left(\left(T^{2} R\right) R\left(R T^{2}\right)\right] \subseteq\left(T^{2} R T^{2}\right] .
\end{aligned}
$$

So, $T \subseteq\left(T^{2} R T^{2}\right] \subseteq(A R A] \subseteq(A]=A$. Hence $A$ is a semiprime bi-hyperideal of $R$.

Theorem 5.6. Let $(R,+, \cdot, \leq)$ be a regular ordered Krasner hyperring. Then, the following statements hold:

(1) $A \cap K_{1} \cap K_{2} \subseteq A K_{1} K_{2}$ for every left hyperideals $K_{1}, K_{2}$ and right hyperideal $A$ of $R$.

(2) $B \cap K_{1} \cap K_{2} \subseteq B K_{1} K_{2}$ for every left hyperideals $K_{1}, K_{2}$ and interior hyperideal $B$ of $R$.

(3) $C \cap K_{1} \cap K_{2} \subseteq C K_{1} K_{2}$ for every left hyperideals $K_{1}, K_{2}$ and bi-hyperideal $C$ of $R$.

(4) $D \cap E \cap K \subseteq D E K$ for every bi-hyperideal $D$, interior hyperideal $E$ and left hyperideal $K$ of $R$. 
Proof. (1): Let $a \in A \cap K_{1} \cap K_{2}$. Since $R$ is regular, there exists $x \in R$ such that $a \leq a x a$. Thus we have $a \leq$ axa $\leq$ ax $($ axa $) \in(A R) K_{1} R K_{2} \subseteq A K_{1} K_{2}$. Therefore, we have $A \cap K_{1} \cap K_{2} \subseteq A K_{1} K_{2}$.

(2): Let $B$ be an interior hyperideal of $R$. By Corollarly 4.5, $B$ is a hyperideal of $R$. The rest of this proof is similar to the proof of (1).

(3): Let $a \in C \cap K_{1} \cap K_{2}$. Since $R$ is regular, there exists $x \in R$ such that $a \leq a x a$. Since $C$ is a bi-hyperideal of $R$, it follows that $a \leq a x a \leq$ $a x(a x a) \leq \operatorname{axax}(a x a) \in(C R C) R K_{1} R K_{2} \subseteq C K_{1} K_{2}$. Therefore, we have $C \cap K_{1} \cap K_{2} \subseteq C K_{1} K_{2}$.

(4): By Corollarly 4.5, $E$ is a hyperideal of $R$. The rest of this proof is similar to the proof of (3).

At the end of the paper, we prove the following theorem.

Theorem 5.7. Let $P$ be a prime left hyperideal of an ordered Krasner hyper$\operatorname{ring}(R,+, \cdot, \leq)$. Then, the set $(P: a)=\{x \in R: x \cdot a \in P\}$ is a prime left hyperideal of $R$, for any $a \in R \backslash P$.

Proof. Since $0 \in(P: a)$, it follows that $(P: a) \neq \emptyset$. Let $x, y \in(P: a)$. Then $x \cdot a \in P, y \cdot a \in P$. Thus we have $(x+y) \cdot a=x \cdot a+y \cdot a \subseteq P$ and $(-x) \cdot a=$ $-(x \cdot a) \in P$. So, $x+y \subseteq(P: a)$ and $-x \in(P: a)$. Now, let $x \in(P: a)$ and $r \in R$. Since $x \cdot a \in P$, it follows that $(r \cdot x) \cdot a=r \cdot(x \cdot a) \in R \cdot P \subseteq P$. So, $r \cdot x \subseteq(P: a)$. Let $x \in(P: a), y \in R$ and $y \leq x$. Then $x \cdot a \in P$ and $y \cdot a \leq x \cdot a$. Since $P$ is a left hyperideal of $R$, it follows that $y \cdot a \in P$. So, $y \in(P: a)$. Hence $(P: a)$ is a left hyperideal of $R$.

Finally, let $A$ and $B$ be any left hyperideals of $R$ such that $A B \subseteq(P: a)$. Then $(A B) a \subseteq P$. Since $P$ is a left hyperideal of $R$, it follows that $(P]=P$. It is easy to see that $(A a]$ and $(B a]$ are left hyperideals of $R$. Thus we have $(A a] \cdot(B a] \subseteq(A(a B) a] \subseteq((A B) a] \subseteq(P]=P$. Since $P$ is a prime left hyperideal of $R$, it follows that $(A a] \subseteq P$ or $(B a] \subseteq P$. Thus $A a \subseteq P$ or $B a \subseteq P$. So, we have $A \subseteq(P: a)$ or $B \subseteq(P: a)$. Therefore, $(P: a)$ is a prime left hyperideal of $R$.

\section{References}

[1] F. W. Anderson, Lattice-ordered rings of quotients, Canad. J. Math., 17 (1965), 434-448.

[2] A. Asokkumar, Derivations in hyperrings and prime hyperrings, Iran. J. Math. Sci. Inform., 8 (2013), 1-13.

[3] A. Asokkumar, Hyperlattice formed by the idempotents of a hyperring, Tamkang. J. Math., 38(3) (2007), 209-215. 
[4] M. Bakhshi and R. A. Borzooei, Ordered Polygroups, Ratio Math., 24 (2013), 31-40

[5] G. Birkhoff, Lattice Theory, Providence, 1969.

[6] T. Changphas and Bijan Davvaz, Properties of hyperideals in ordered semihypergroups, Ital. J. Pure Appl. Math., 33 (2014), 425-432.

[7] J. Chvalina, Commutative hypergroups in the sence of Marty and ordered sets, General algebra and ordered sets (Horn Lipova, 1994), 19-30.

[8] J. Chvalina and J. Moucka, Hypergroups determined by orderings with regular endomorphism monoids, Ital. J. Pure Appl. Math., 16 (2004), 227-242.

[9] P. Corsini, Prolegomena of Hypergroup Theory, Second edition, Aviani editor, Tricesimo, 1993.

[10] P. Corsini and V. Leoreanu-Fotea, Applications of Hyperstructure Theory, Advances in Mathematics, Kluwer Academic Publication, Dordrecht, 2003.

[11] B. Davvaz, Isomorphism theorems of hyperrings, Indian J. Pure Appl. Math., 35(3) (2004), 321-331.

[12] B. Davvaz, Polygroup Theory and Related Systems, World Scientific Publishing Co. Pte. Ltd., Hackensack, NJ, 2013.

[13] B. Davvaz and A. Koushky, On hyperring of polynomials, Ital. J. Pure Appl. Math., 15 (2004), 205214.

[14] B. Davvaz and V. Leoreanu-Fotea, Hyperring Theory and Applications, International Academic Press, Palm Harbor, USA, 2007.

[15] B. Davvaz, P. Corsini and T. Changphas, Relationship between ordered semihypergroups and ordered semigroups by using pseudoorder, European J. Combin., 44 (2015), 208-217.

[16] R. A. Good and D. R. Hughes, Associated groups for a semigroup, Bull. Amer. Math. Soc., 58 (1952), 624-625.

[17] D. Heidari and B. Davvaz, On ordered hyperstructures, Politehn. Univ. Bucharest Sci. Bull. Ser. A Appl. Math. Phys., 73(2) (2011), 85-96.

[18] S. Hoskova, Representation of quasi-order hypergroups, Glob. J. Pure Appl. Math., 1 (2005), 173-176. 
[19] S. Hoskova, Upper order hypergroups as a reflective subcategory of subquasiorder hypergroups, Ital. J. Pure Appl. Math., 20 (2006), 215-222.

[20] N. Kehayopulu, Note on interior ideals, ideal elements in ordered semigroups, Sci. Math. 2, No. 3, (1999), 407-409.

[21] N. Kehayopulu, S. Lajos and M. Tsingelis, On intra-regular ordered semigroups, Pure Math. Appl., 4 (1993), 317-327.

[22] M. Krasner, A class of hyperrings and hyperfields, Internat. J. Math. Math. Sci., 2 (1983), 307-312.

[23] V. Leoreanu-Fotea, Canonical n-ary hypergroups, Ital. J. Pure Appl. Math., 24 (2008), 247-254.

[24] F. Marty, Sur une generalization de la notion de groupe, 8th Congress of Mathematics in Scandenaves, Stockholm, (1934), 45-49.

[25] J. Mittas, Hypergroups canoniques, Math. Balkanica, (1972), 165-179.

[26] A. Nakassis, Expository and survey article recent result in hyperring and hyperfield theory, Internet. J. Math Math. Sci., 11(2) (1988), 209-220.

[27] M. Shabir and N. Kanwal, Prime bi-ideals in semigroups, Southeast Asian Bull. Math., 31 (2007), 757-764.

[28] S. Spartalis, A class of hyperrings, Rivista Mat. Pura Appl. 4 (1989), 56-64.

[29] T. Vougiouklis, Hyperstructures and Their Representations, Hadronic Press, Inc., 115, Palm Harber, USA, 1994.

S. OMIDI,

Department of Mathematics,

Yazd University,

Yazd, Iran.

Email: omidi.saber@yahoo.com

B. DAVVAZ,

Department of Mathematics,

Yazd University,

Yazd, Iran.

Email: davvaz@yazd.ac.ir, bdavvaz@yahoo.com

P. CORSINI,

Department of Civil Engineering and Architecture

Via delle Scienze 206, 33100 Udine, Italy

E-mail: piergiulio.corsini@uniud.it 